\title{
Ultra-Reiz Current Appears to be More Effective Modality than Interferential for People with Knee Osteoarthritis: A Randomized Block Clinical Trial
}

\author{
Mahnaz Ahangari ${ }^{1 *}$, Amin Norouzi Fashkhami ${ }^{2}$, Hamid Zinalpoor ${ }^{3}$, Hamed Ahangari ${ }^{4}$, \\ Mohamad Reza Minator Sajjadi ${ }^{5}$
}

${ }^{1}$ Taleghani Hospital, Research Development Committee, Department of physical therapy, Shahid Beheshti University of Medical Sciences

${ }^{2}$ Physical therapy department, Shahid Beheshti University of Medical Sciences Tehran, Iran ${ }^{3}$ College of Rehabilitation, Shahid Beheshti University of Medical Sciences Tehran, Iran ${ }^{4}$ Private sector in Washington, USA ${ }^{5}$ Department of orthopedic surgery, Taleghani Hospital, Shahid Beheshti University of Medical Sciences, Tehran, Iran

*Corresponding Author: Mahnaz Ahangari; Taleghani Hospital, Research Development Committee, Department of physical therapy, Shahid Beheshti University of Medical Sciences; mahnaz ahangari@sbmu.ac.ir

Received : 2020-07-05

Accepted : 2020-10-27

Published : 2020-12-01

\begin{abstract}
Introduction: Today, increased degenerative disease of the knee, which reduces the efficiency of the activity and quality of life, is one of the most common causes of many population complaints. It is important to properly plan and carry out non-invasive and nonpharmacological therapies to treat the pain and its consequences. The question of which of methods effectively relieves pain, improve function and efficiently increases life quality led to a comparison of two methods, interferential and ultra-reiz (Träbert) currents therapy.

Methods: Randomized Block clinical trial carried out at Taleghani general hospital Shahid Beheshti University of Medical Sciences from November 2017 to February 2019. The study included 104 patients of both sexes, aged 38-74 years, with knee osteoarthritis. They were selected based on exclusion and inclusion criteria. The patients were randomly assigned to two groups: $A$ and $B$. Group $A, n$ : 52 patients, were received ultra-reiz current, and group $B, n$ : 52 patients, were subjected to interferential current. Parameters
\end{abstract}

assessment were evaluated by the Knee Injury and 0steoarthritis Outcome Score (KOOS) questionnaire with 5 domains: pain, symptoms, ADL, sports/recreational activity, and quality of life. Data were collected at the 1st session and at the end of treatment (at least three times a week). The obtained results were subject to statistical analysis.

Results: Data were analyzed in SPSS software by using paired and two independent sample t-test. The mean scores before and after treatment with $\mathrm{p}$-value range $0.002 \sim 0.764$ showed that there were differences in pain, other symptoms, ADL, sport/recreational activity, and quality of life status between ultra-reiz and IFT groups, but the results were statistically more significant in the ultra-reiz group ( $p<0.0001)$.

Conclusions: Our study showed that ultra-reiz and IFT were effective for patients with knee $0 \mathrm{~A}$, but ultra-reiz became a more effective modality than IFT in OA patients.

Keywords: ultra-reiz, interferential current, knee osteoarthritis

Cite this Article: Ahangari, M., Fashkhami, A.N., Zinalpoor H., Ahangari, H., Sajjadi, M.R.M. 2020. Ultra-Reiz Current Appears to be More Effective Modality than Interferential for People with Knee Osteoarthritis: A Randomized Block Clinical Trial. Physical Therapy Journal of Indonesia 1(2): 29-34.

\section{INTRODUCTION}

Osteoarthritis (OA) is one of common degenerative conditions leads to disability, particularly in the older population. In the developed countries it has been taken into account as the most joint problem and a leading cause of chronic pain, mainly owing to knee and/or hip OA. ${ }^{1}$ Knee OA represents a significant burden on healthcare provision with remarkable health and welfare costs. ${ }^{2}$ Although it may have various and different etiologies but its biologic, morphologic, and clinical findings are similar. ${ }^{3}$ The number of patients suffering from $\mathrm{OA}$ is likely to increase because of increasing elderly population and the obesity epidemic. ${ }^{4}$ The prevalence rates of $\mathrm{OA}$ varies in different countries, which possibly contributes by differences in race, culture, lifestyle. According to numerous investigations age, sex, weight, occupation, sports and recreational activities have significant role as risk factors in the progression of OA. ${ }^{5}$ The clinical features for diagnosis of OA includes a number of symptoms and signs, three latent symptoms are knee pain, morning stiffness, and function loss and three latent signs are crepitus, decrease ROM, and bony enlargement. ${ }^{6}$ A main risk factor and one of pathogenic forces in $\mathrm{OA}$ is mechanical stress which has been detected as a significant factor in causing the inflammatory and catabolic process. ${ }^{7}$

Inflammatory response of joint cartilage is demonstrated as erosion or focal loss of cartilage and activity of osteoblast that is characterized by osteophytosis. ${ }^{8}$ In affected population some symptoms of OA namely muscle weakness, joint stiffness, and chronic pain which are serious risk factors for restricted motion and results in impaired quality of life. ${ }^{9}$ Treatment plan for knee OA should be based on pain relief, joint mobility improvement 
and limit function impairment, and it includes both surgical and conservative options. Surgical methods include various types of arthroscopic interventions, knee arthroplasty, and osteotomy. Non-operative options can be achieved by nonpharmacological and pharmacological methods. Non-operative treatment is usually appropriate for knee OA patients in grades 1-3, which are early stages of OA. ${ }^{10}$ To achieving the goals of pain controlling, discomfort, and disability caused by the knee OA condition, through noninvasive and non-pharmacological methods, physical therapy with various techniques is considered as an effective treatment and among the variety of the techniques for pain and its outcomes control, electrotherapy is an effective and appropriate method. ${ }^{11}$

The purpose of physical therapy is to reduce pain, maintaining joint mobility, and improving muscle strength. In a meta-analysis, Page et al. ${ }^{12}$ declared that in individuals affected with knee OA, there is strong evidence to indicate that physical therapy interventions can improve the knee's pain and functions. Electrotherapy involves a variety of modalities to treat various diseases. Ultra-reiz is a low frequency rectangular galvanic type of current that has been used for improving knee's pain and functions in knee osteoarthritis patients, lateral humeral epicondylitis, and whiplash injury. ${ }^{13-15}$ The current leads to a rise of temperature both locally and segmentally in the stimulation site, which thermal reactions depends on the distance from the treated area. ${ }^{16}$

Interferential therapy (IFT) is a common physiotherapeutic treatment modality used in physical therapy clinics. IFT effectively reduces pain and improves physical function ${ }^{17}$ and when it combined with exercise demonstrated a positive interaction effect in pain reduction, decreased medication intake, and improve knee functions in patients with knee OA. ${ }^{18}$

This study aimed to gather evidence of noninvasive methods and evaluate the effects of ultra-reiz and IFT currents and the comparison of them on pain and function in patients with knee $\mathrm{OA}$. We combined an exercise program with these two modalities to maximize any effect on pain and joint stiffness and disability and function.

\section{METHODS}

\section{Participants and data collection}

This randomized block clinical trial was carried out at the physical therapy department, Taleghani General Hospital, Shahid Beheshti University of Medical Sciences Tehran, Iran from November 2017 to February 2019. The study included 104
Subjects of 37 men and 67 women, aged 38-78 years with knee osteoarthritis.

Participants were selected based on exclusion and inclusion criteria. The inclusion criteria were patients with chronic pain at least for three months, limited in ADL and functional ability, being at stages 2 and 3 of knee osteoarthritis (mild to moderate knee osteoarthritis, based on American College of Rheumatology criteria), experience frequent pain during movement, and without anti-inflammation medication intake. The exclusion criteria were neuropathic disorder, nerve injury, rheumatologic disease, autoimmune disease, history of knee fracture and surgery, intra-articular corticosteroid injection, structural giving way, contraindications for electrotherapy, heart disease or having electrical implants such as a pacemaker, malignancies, and epilepsy.

The study protocol has been approved by the ethics committee of Shahid Beheshti University of Medical Sciences, and participants' consent was taken before the study. Before starting treatment, skin evaluation is considered to be healthy, and no scratches on it. All patients were randomly assigned to ultra-reiz $(\mathrm{n}=52)$ and IFT $(\mathrm{n}=52)$ groups. Study outcomes were evaluated by the KOOS questionnaire, which was collected before the 1st session and the end of the 10th session treatment at least three times a week. When the patients reported that the pain was reduced, they underwent an exercise program for the rest of duration of treatment, every day for 3sets and 10 repetitions.

\section{Interventions \\ Ultra-reiz}

Ultra-reiz (Enraf-Nonius is made in the Netherlands) is rectangular impulses lasting $2 \mathrm{~ms}$ include a $5 \mathrm{~ms}$ pause between single impulses, causing to approximately $143 \mathrm{~Hz}$ from the mathematical calculations. The intensity of the current depends on the patient's tolerance. Ultrareiz currents are mainly for improving pain in the locomotor system area and as a selective stimulation of thick fibers. ${ }^{19}$ The increasing amplitude makes it necessary to use thick moist viscose sponges (minimum thickness $1 \mathrm{~cm}$ ) and medium-sized electrodes. In local treatment, electrodes are placed at a distance of $3 \mathrm{~cm}$ between each other. The cathode should be placed at the pain site. Before local treatment, segmental treatment is initially performed on the vertebral column to stimulate the vegetative nervous system. One electrode is placed at the lumbosacral junction and the other on the dorsal of the lumbar vertebra. The current intensity is slowly increased in the first few minutes 
as patients get used to the sensation from mild to patient's tolerance level. ${ }^{13}$ Treatment time was 10 minutes. After treatment was completed in the lumbar region, electrodes were placed locally on either side of the knee joint, for another 10 minutes.

\section{IFT}

IFT (Enraf-Nonius is made in the Netherlands) predominately excites large-diameter nerve fibers and reduces the transmission of nociceptive signals through small-diameter nerve fibers to the spinal dorsal horn by presynaptic inhibition, thus achieving pain modulation in the higher center. ${ }^{20}$ IFT is associated with lower skin resistance, comfortable \& tolerance, and deep penetration. Two medium frequency currents can be given out of phase; these currents interrupt with each other inside tissues when the currents cross. The resultant amplitude-modulated interference that have been reported to stimulate analgesic effect produce in beat frequencies of between 1 and $250 \mathrm{~Hz}^{21}$ The patients were treated with quadripolar vector scan electrode technique, covering quadriceps muscle and knee joint using the beat frequency of $100 \mathrm{~Hz}$ and carrier frequency of $4000 \mathrm{~Hz}$. The intensity was adjusted according to patients' tolerance, strong but comfortable. Treatment time was 20 minutes, and electrodes were placed locally on both sides of the knee joint.

\section{Exercises}

Patients reported that the pain was reduced from the third session, then after the last treatment session, all individuals in the two groups, underwent in an exercise program that include isometric exercise, terminal extension in close chain, and straight leg raise of 10 repetitions for 3 sets.

\section{Statistical analysis}

Continuous data of KOOS's pain, symptoms, ADL, sports/recreational activity, and quality of life were entered into SPSS software, version 25 (IBM Corporation, Armonk, NY) for statistical analysis. The paired T-test was used to examine whether data between the pre-test and post-test were statistically significant difference in the two groups. The independent T-test was performed to evaluate mean changes of ultra-reiz and IFT groups whether they were significantly difference. A $p$-value of $<0.05$ in the results was considered statistically significant.

\section{RESULTS}

To achieve the goal of study, the mean variables of both groups should not be significantly different before intervention, which assumed the intervention groups have equal baseline characteristics. Based on the findings no significant difference was found in the mean of variables between ultra-reiz and IFT groups before the intervention.

The KOOS scores in the ultra-reiz group before treatment were $22.8 \sim 61.5$ and after treatment ranged were 51.4 86.3, whereas in the IFT group before treatment were $22.3 \sim 55.0$ and after treatment

Table 1. Comparisons of the Knee Injury and Osteoarthritis Outcome Score (KOOS) domains at the baseline and their changes over the 10-times interventions among the ultra-reiz and interferential therapy (IFT) groups

\begin{tabular}{|c|c|c|c|c|c|}
\hline KOOS & $\begin{array}{l}\text { At baseline } \\
\text { Mean } \pm \text { SD }\end{array}$ & $\begin{array}{c}\text { After interventions } \\
\text { Mean } \pm \text { SD }\end{array}$ & p-value & $\begin{array}{l}\text { Changes } \\
\text { Mean } \pm \text { SD }\end{array}$ & p-value \\
\hline \multicolumn{6}{|l|}{ Pain } \\
\hline Ultra-reiz & $52.5 \pm 19.5$ & $81.8 \pm 9.4$ & 0.045 & $29.41 \pm 0.1$ & \multirow{2}{*}{$<0.000$} \\
\hline IFT & $46.8 \pm 14.5$ & $65.8 \pm 10.7$ & 0.314 & $19.0 \pm 3.8$ & \\
\hline \multicolumn{6}{|l|}{ Symptoms } \\
\hline Ultra-reiz & $61.5 \pm 18.0$ & $86.2 \pm 10.1$ & 0.403 & $24.7 \pm 7.9$ & \multirow{2}{*}{$<0.000$} \\
\hline IFT & $55.0 \pm 19.4$ & $69.9 \pm 16.4$ & 0.000 & $14.9 \pm 3.0$ & \\
\hline \multicolumn{6}{|l|}{$\mathrm{ADL}$} \\
\hline Ultra-reiz & $56.6 \pm 19.5$ & $86.3 \pm 8.3$ & 0.181 & $29.7 \pm 11.2$ & \multirow{2}{*}{$<0.000$} \\
\hline IFT & $51.8 \pm 17.2$ & $67.5 \pm 13.3$ & 0.002 & $15.7 \pm 3.9$ & \\
\hline \multicolumn{6}{|l|}{ Sport/Rec } \\
\hline Ultra-reiz & $24.3 \pm 23.7$ & $59.3 \pm 15.5$ & 0.074 & $35.0 \pm 8.2$ & \multirow{2}{*}{$<0.000$} \\
\hline IFT & $22.3 \pm 20.0$ & $30.5 \pm 21.9$ & 0.150 & $8.1 \pm 1.9$ & \\
\hline \multicolumn{6}{|l|}{ QOL } \\
\hline Ultra-reiz & $22.8 \pm 12.3$ & $51.4 \pm 18.7$ & 0.764 & $28.6 \pm 6.8$ & \multirow{2}{*}{$<0.000$} \\
\hline IFT & $26.0 \pm 12.2$ & $38.6 \pm 12.7$ & 0.210 & $12.6 \pm 0.0$ & \\
\hline
\end{tabular}

SD, standard deviation 


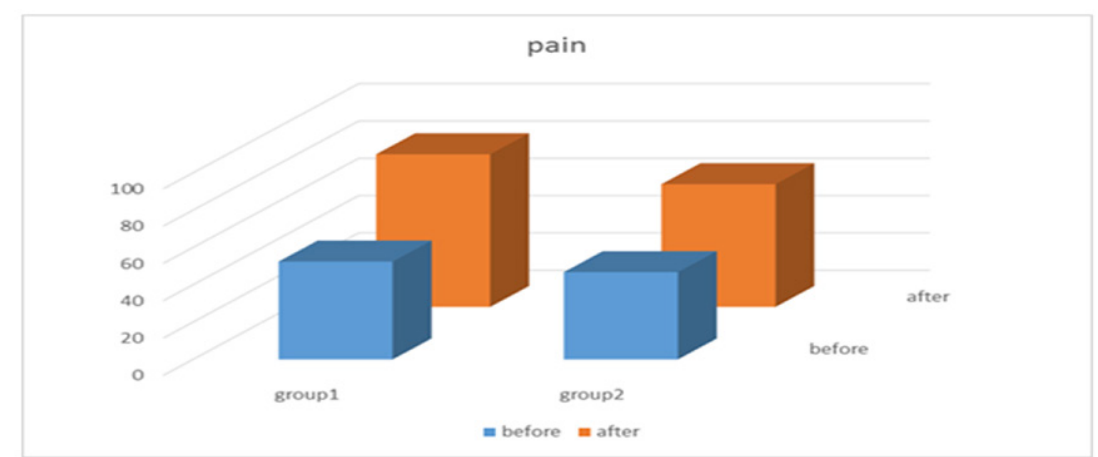

Figure 1. Mean pain variable in groups 1 and 2 before and after treatment

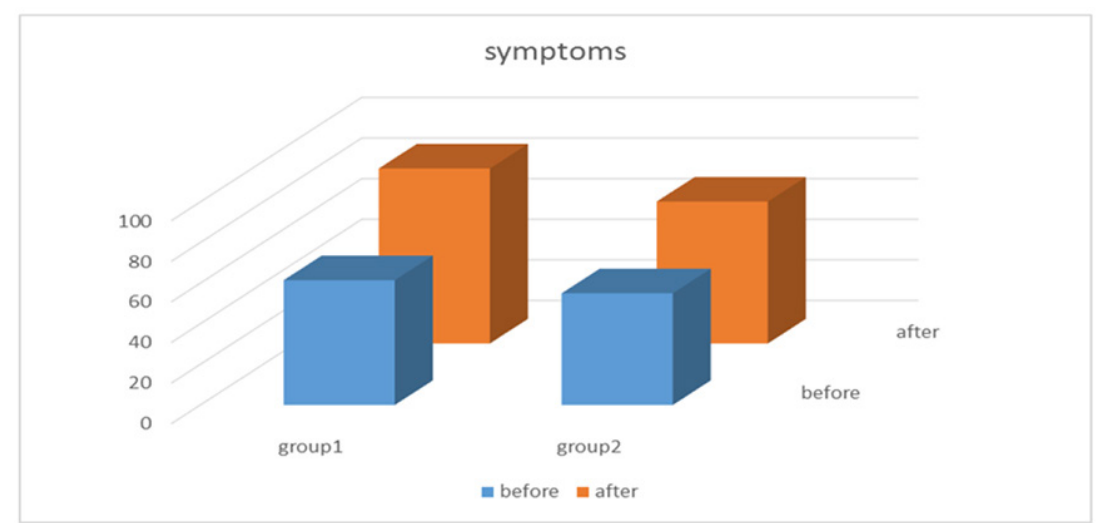

Figure 2. Mean symptoms variable in groups 1 and 2 before and after treatment

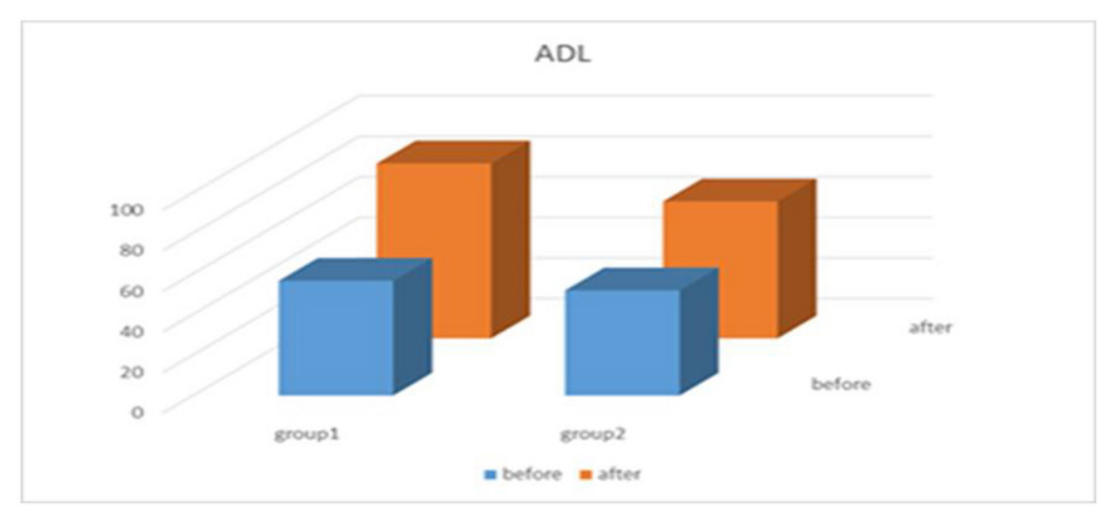

Figure 3. Mean ADL variable in groups 1 and 2 before and after treatment

were 30.5 69.9. There were differences between pre-test and post-test scores in both intervention groups (p-value: ultra-reiz $=0.045 \sim 0.764$, IFT $=$ $<0.0001 \sim 0.314$ ) (Table 1). Figures 1-5 showed means of variables in the ultra-reiz and IFT before and after interventions.

There were significant differences in mean changes of KOOS's domains between ultra-reiz and IFT groups, whereas the ultra-reiz intervention had bigger changes than the IFT intervention.

\section{DISCUSSION}

The goal of knee OA intervention is to improve the patients' pain, functions, and quality of life. ${ }^{6}$ Physical therapy is one of the reliable method in conservative interventions for pain control and functional improvement. ${ }^{22}$ This was a randomized block clinical trial, which aimed to evaluate the efficacy of ultra-reiz and IFT as electrotherapeutic modalities in patients with knee OA and parameters measured by the KOOS questionnaire.

The pathophysiology of pain, symptom, and structural change of cartilage are complex. Elements related to the knee which are rich innervated are subchondral bone, periosteum, ligaments, muscles, synovium, and joint capsule, being the source of nociception in OA. Diagnostic magnetic resonance imaging (MRI) has shown some significant effects in joint structure for instance bone marrow lesions, sub-articular bone erosion, synovitis, and effusion have been associated with the knee pain. Of 351 persons with the knee pain, $272(77.5 \%)$ had bone marrow, but only 15 out of 50 (30\%) among persons without no knee pain had bone marrow. ${ }^{23}$ Decreased blood flow and elevated interosseous pressure lead to bone angia which along with periostitis related to osteophyte information and subchondral microfractures provide bone-derived pain.

In arthritis, pain-producing biochemical elements such as prostaglandins, histamine, bradykinin, vasoactive intestinal peptide, substance $\mathrm{P}$, lactic acid, and calcitonin gene-related peptide (CGRP) are spread out into the joint. These substances decrease the firing threshold of nociceptors on the knee joint, which induced the noxious- and non-noxious-pain. ${ }^{24}$

Hajizadeh et al. ${ }^{25}$ suggested that the skin's electrical stimulation increases local blood flow through two mechanisms, releasing some vasodilator neurotransmitters nerve endings and removing sympathetic tone. Besides, electrical stimulation can alter bone hydrodynamics and subsequently improve venous return, reducing intra-osseous pressure and relieving pain. In this study, ultra-reiz effectively improved patients' pain, function, and quality of life, possibly due to increasing circulation that releases subchondral bone congestion, decreasing the intraosseous pressure, relieving pain, and improved mobility and function. ${ }^{13,14}$ It has been demonstrated that improved the circulation to the knee joint washed out the noxious biochemical elements such was prostaglandin and bradykinin. ${ }^{13}$ Ultra-reiz intervention improved circulation that released the congestion of subchondral bone, which decreased 


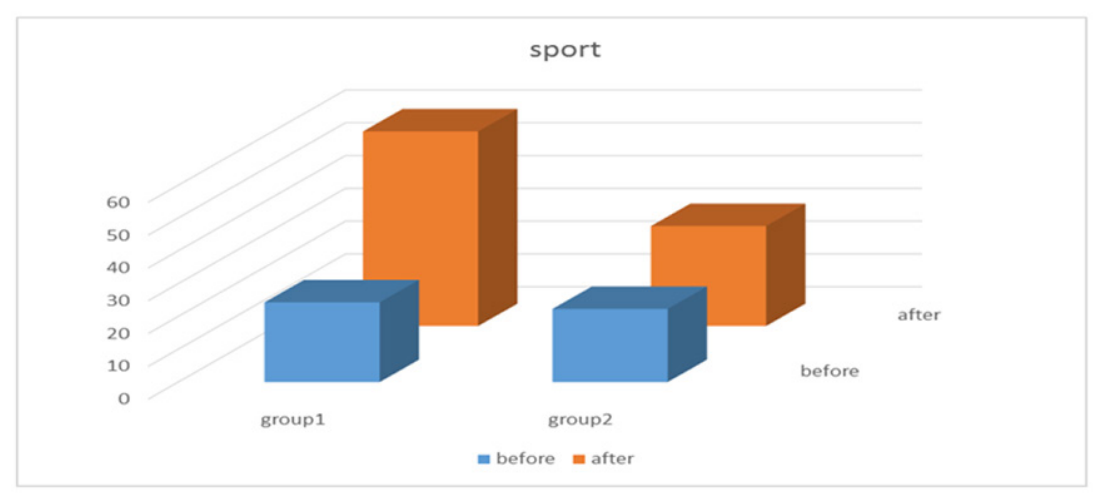

Figure 4. Mean sport variable in groups 1 and 2 before and after treatment

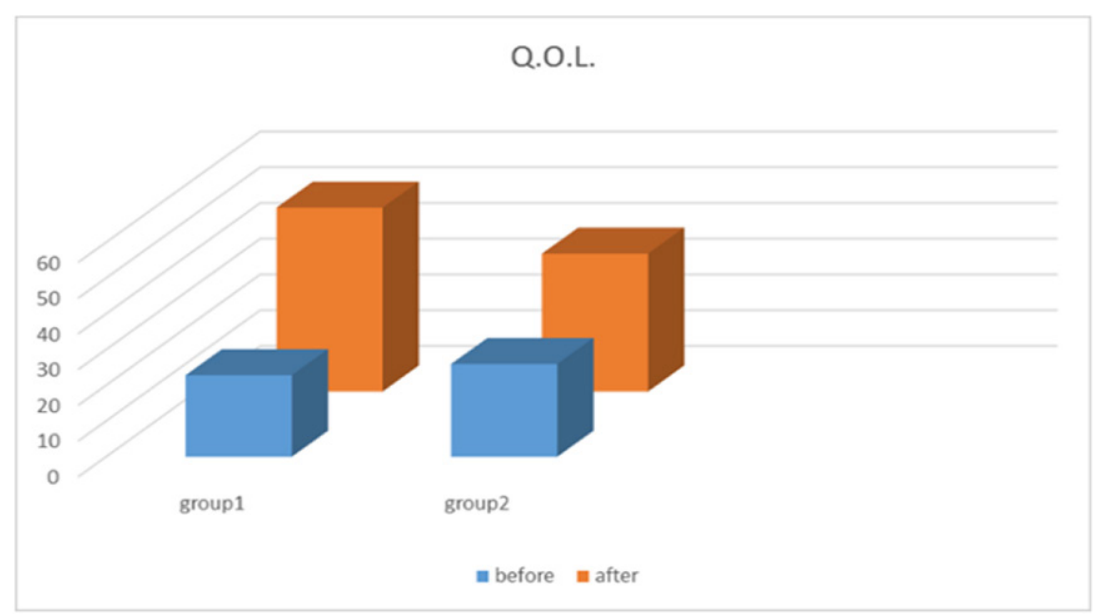

Figure 5. Mean of quality of life variable in groups 1 and 2 before and after treatment

\section{CONCLUSION}

According to our findings, it can be concluded that ultra-reiz and IFT have proved to be effective physical modalities in treating patients with knee OA. Comparison between both interventions demonstrated that ultra reiz current therapy was more effective in all domains of the KOOS questionnaire than IFT.

\section{CONFLICT OF INTEREST}

There is no conflict of interest

\section{FUNDING}

N/A

\section{AUTHOR CONTRIBUTIONS}

MA conceived the study design, data collections, data analysis, and drafted the paper, and ANF, HZ, HA, and MRMS conducted data collections, data analysis, and drafted the

\section{REFERENCES}

1. Grazio S, Balen D. Obesity: risk factor and predictor of osteoarthritis. Lijec Vjesn. 2009;131(1-2):22-6.

2. Hinman RS, Hunt MA, Creaby MW, Wrigley TV, McManus FJ, Bennell KL. Hip muscle weakness in individuals with medial knee osteoarthritis. Arthritis Care Res (Hoboken). 2010;62(8):1190-3.

3. MacLean $\mathrm{CH}$. Quality indicators for the management of osteoarthritis in vulnerable elders. Ann Intern Med. 2001;135(8_Part_2):711-21.

4. Zhang Y, Jordan JM. Epidemiology of osteoarthritis. Clin Geriatr Med. 2010;26(3):355-69.

5. He Q-Q, Zhang J-F. Prevalence of osteoarthritis and association between smoking patterns and osteoarthritis in China: a cross-sectional study. Frontiers of Nursing. 2018;5(2):111-8.

6. Zhang W, Doherty M, Peat G, Bierma-Zeinstra M, Arden N, Bresnihan B, et al. EULAR evidence-based recommendations for the diagnosis of knee osteoarthritis. Ann Rheum Dis. 2010;69(3):483-9.

7. Pulsatelli L, Addimanda O, Brusi V, Pavloska B, Meliconi R. New findings in osteoarthritis pathogenesis: therapeutic implications. Ther Adv Chronic Dis. 2013;4(1):23-43.

8. Esser S, Bailey A. Effects of exercise and physical activity on knee osteoarthritis. Current pain and headache reports. 2011;15(6):423-30.

9. Liikavainio T, Lyytinen T, Tyrväinen E, Sipilä S, Arokoski JP. Physical function and properties of quadriceps femoris muscle in men with knee osteoarthritis. Arch Phys Med Rehabil. 2008;89(11):2185-94.

10. Vaishya R, Pariyo GB, Agarwal AK, Vijay V. Non-operative management of osteoarthritis of the knee joint. Journal of Clinical Orthopaedics and Trauma. 2016;7(3):170-6.

11. Dohnert MB, Bauer JP, Pavão TS. Study of the effectiveness of interferential current as compared to transcutaneous electrical nerve stimulation in reducing chronic low back pain. Revista Dor. 2015;16(1):27-31. 
12. Page CJ, Hinman RS, Bennell KL. Physiotherapy management of knee osteoarthritis. Int J Rheum Dis. 2011;14(2):145-51.

13. Sen S, Kumar TRN, Rau SS. Effects of ultra reiz current and tens on pain and Functional ability in older patients with osteoarthritis Knee. Int J Physiother Re. 2013;1(4):171-6.

14. Fornés IP, Tauriaux OP, Mayet EQ, Calderón MC, Suárez MJ. Effectiveness of the segmental treatment with Trabert current in patient with epicondylitis external humeral. MediSan. 2015;19(06):747-55.

15. Hendriks O. Ultra-reiz current as an adjunct to standard physiotherapy treatment of the acute whiplash patient. Physiotherapy Ireland. 1996;17:3-7.

16. Hanna A-B, Gieremek K, Kubacki J, Grygorowicz M. Local and segmentary effects of Träbert's current as observed in thermovision imaging. Fizjoterapia Polska. 2005;5(1):1-7.

17. Eftekharsadat B, Babaei-Ghazani A, Habibzadeh A, Kolahi B. Efficacy of action potential simulation and interferential therapy in the rehabilitation of patients with knee osteoarthritis. Ther Adv Musculoskelet Dis. 2015;7(3):6775.

18. Buenavente M, Gonzalez-Suarez C, Lee-Ledesma M, Liao L. Evidence on the effectiveness of interferential current therapy in the treatment of knee osteoarthritis: a metaanalysis. OA Arthritis. 2014;2(1):1-9.

19. Träbert currents. In: Rovenský J, Payer J, editors. Dictionary of Rheumatology. Vienna: Springer Vienna; 2009. p. 216-.

20. Watson T. Electrotherapy E-Book: evidence-based practice: Elsevier Health Sciences; 2008.
21. Jorge Sr, Parada CA, Ferreira SrH, Tambeli CuH. Interferential therapy produces antinociception during application in various models of inflammatory pain. Phys Ther. 2006;86(6):800-8.

22. Kumar SP, Saha S. Mechanism-based classification of pain for physical therapy management in palliative care: A clinical commentary. Indian J Palliat Care. 2011;17(1):80.

23. Felson DT, Chaisson CE, Hill CL, Totterman SM, Gale ME, Skinner KM, et al. The association of bone marrow lesions with pain in knee osteoarthritis. Ann Intern Med. 2001;134(7):541-9.

24. Hunter DJ, McDougall JJ, Keefe FJ. The symptoms of osteoarthritis and the genesis of pain. Rheumatic Disease Clinics of North America. 2008;34(3):623-43.

25. Hajizadeh S, Khoshbaten A, Asgari A. Mechanisms of blood flow increase induced by electrical stimulation. Medical Journal of The Islamic Republic of Iran (MJIRI). 2000;13(4):279-82.

26. Tabasam G, Johnson MI. The use of interferential therapy for pain management by physiotherapists. International journal of therapy and rehabilitation. 2006;13(8):357-64.

27. Adedoyin RA, Olaogun MO, Fagbeja OO. Effect of interferential current stimulation in management of osteoarthritic knee pain. Physiotherapy. 2002;88(8):493-9.

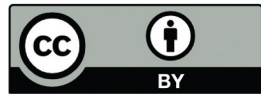

This work is licensed under a Creative Commons Attribution 